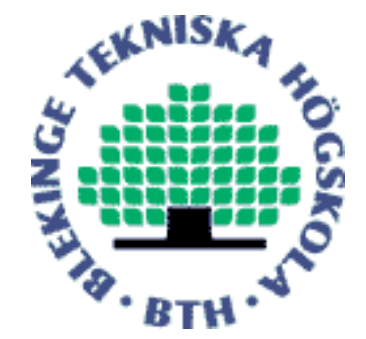

Copyright (C) 2012 IEEE.

Citation for the published paper:

Cognitive Radio Spectrum Decision Based on Channel Usage Prediction

Yong Yao, Said Rutabayiro Ngoga, Adrian Popescu

Eighth Euro-NF Conference on Next Generation Internet (NGI)

2012 Karlskrona

This material is posted here with permission of the IEEE. Such permission of the IEEE does not in any way imply IEEE endorsement of any of BTH's products or services Internal or personal use of this material is permitted. However, permission to reprint/republish this material for advertising or promotional purposes or for creating new collective works for resale or redistribution must be obtained from the IEEE by sending a blank email message to pubs-permissions@iee.org.

By choosing to view this document, you agree to all provisions of the copyright laws protecting it. 


\title{
Cognitive Radio Spectrum Decision Based on Channel Usage Prediction
}

\author{
Yong Yao, Said Rutabayiro Ngoga, and Adrian Popescu \\ School of Computing, Blekinge Institute of Technology, 37179 Karlskrona, Sweden \\ Email: \{yya, srn, apo\}@bth.se
}

\begin{abstract}
The paper is about a new strategy suggested for spectrum decision in Cognitive Radio (CR) networks. By jointly considering sensing error, Secondary Users (SUs) competition and SUs transmission collision, a new parameter called Channel Usage State (CUS) is introduced. For a particular channel, we predict the respective probabilities of occurrence of CUS states by using the LeZi-update scheme. We also adopt a fuzzy comparison algorithm to combine the prediction results as a joint value. The largest joint value is associated with the most available channel for access by SUs in the near future. By comparing with random channel access, the suggested strategy can improve SUs transmission throughput. This is demonstrated by the simulation evaluations.
\end{abstract}

Index Terms-Cognitive radio, imperfect sensing, transmission collision, LeZi-update scheme.

\section{INTRODUCTION}

Cognitive Radio (CR) networks are a technology advanced to solve the problem of spectrum scarcity [1]. In CR networks, the spectrum bands (i.e., radio channels) are exclusively reserved for use by licensed users, called Primary Users (PUs). The available channels not being used by PUs can be temporarily used by unlicensed users, called Secondary Users (SUs) [2]. Identification of channel availability is usually done by spectrum sensing [3]. Since there may exist multiple available channels at a time moment, SU is faced with the task to decide on which available channel should be selected. The selection process is often referred to as spectrum decision with the aim to optimize the transmission performance of SUs.

Due to channel availability varying over time, one approach for optimal spectrum decision is to learn from channel occupancy statistics of PUs. For example, the authors of [4] suggest a continuous-time Markov chain based modeling approach to study the arrival and departure processes of both PUs and SUs. However, there are three problems existing in statistics based spectrum decision. They are sensing error, SUs competition and SUs transmission collision, as described in the following. First, the performing of spectrum sensing may be imperfect in practical implementation. The imperfect sensing may create errors in terms of overlook and misidentification of channel availability [5]. Second, multiple SUs may select the same channel with respect to the same statistical information. As a result, they are likely to compete for the channel utilization in the selected channel. Third, SUs competition can be alleviated by using Carrier Sense Multiple Access with Collision Avoidance (CSMA/CA) based protocol [6]. Under such protocol, one or more SUs may have the same smallest backoff time in the same selected channel. In this case, their transmissions collide with each other when backoff time expires.

The above described problems are widely reported in recent studies. However, in most of them, the effects on spectrum decision are separately investigated. For instance, the authors of [5] suggest a decentralized MAC protocol for SUs in the presence of sensing errors. While, this protocol does not consider the SUs competition and transmission collision. In [7], the authors report on the impact of sensing errors and SUs transmission collision, but they do not give the detailed solutions to overcome the impacts. In [8], under the assumption of perfect sensing, the authors report on an overcrowded case that a large number of SUs simultaneously use the same channels.

Given a realistic CR networks, all three problems, i.e., sensing errors, SUs competition and SUs transmission collision, can not be avoided. Therefore, they need to be jointly taken into consideration in developing practical yet effective spectrum decision strategy. To our best knowledge, there has been little studies so far along with this line.

In this paper, an infrastructure based CR network is considered, where multiple SUs employ CSMA/CA based protocol to share multiple channels. The issue of transmission throughput of SUs is addressed by jointly considering sensing errors, SUs competition and SUs transmission collision. Based on this, a new parameter called Channel Usage State (CUS) is suggested to characterize the channel utilization by PUs and SUs. By using the LeZi-update scheme [9], the uncertainty associated with future CUS is predicted based on CUS history. By using a fuzzy comparison procedure, the joint value of prediction results is computed, and it indicates the numerical channel availability.

The rest of the paper is organized as follows. Section II describes the system model. The definition of CUS and the building of CUS history are presented in Section III. The implementation of predicting CUS is presented in Section IV. The CUS prediction based spectrum decision is presented in Section V. The simulation results are discussed in Section VI. Finally, we conclude the paper in Section VII.

\section{System MODEL}

\section{A. Network Model}

We consider a CR network consisting of $M$ radio channels, each with label $c_{1}, c_{2}, \ldots, c_{M}$. Every channel has an identical amount of bandwidth, which is denoted by $B$. At any specific 


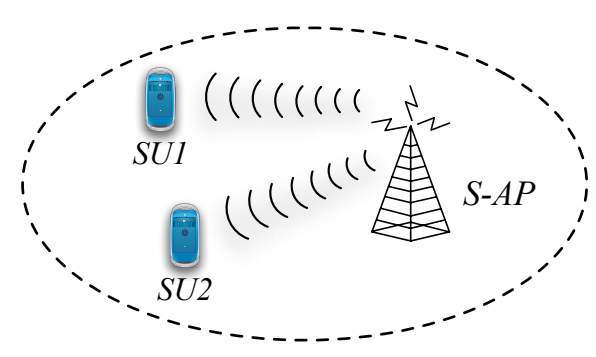

Fig. 1. Example of infrastructure based CR network.

time moment, every channel can be only used by one user, namely, a PU or a SU.

The activity of PUs is assumed to use a synchronous timeslotted basis, i.e., in every slot PU is either present or absent in a channel during the whole slot duration. The length of every slot identically equals $\delta$ in time domain. Moreover, the channel occupancy by PUs is assumed to follow a two-state BUSYFREE Markov process. State BUSY means the event that the PU is occupying a channel for one or more consecutive slots. Similarly, state FREE means the event that there is no PU in a channel for one or more consecutive slots. The time periods of the two states are integer times of $\delta$. They are also assumed to be exponentially distributed with mean values $1 / \mu_{p}$ and $1 / \lambda_{p}$ for states BUSY and FREE, respectively.

In the network, there exist $N$ SUs labeled by $s_{1}, s_{2}, \ldots, s_{N}$. The channel availabilities are assumed to be spatially invariant for all $N$ SUs. In other words, if SUs can do perfect sensing on a channel within a PU slot, the sensing result, i.e., PU being present or absent, is the same at every SU. We also assume that there is a Secondary-Access Point (S-AP) in the network. $\mathrm{S}$-AP can be like a base station or a support node [10]. In addition, each of the $N$ SUs always wants to transmit data to S-AP via an idle channel, as shown in Fig. 1. By an idle channel, we mean the channel is not being used by any PU or SU at a time moment.

\section{B. SU Activity Model}

By communicating with S-AP, the activity of SUs can be synchronized with PUs. As a result, all SUs operate in a time-slotted basis, which has an uniform slot length $\delta$ same with PUs. At the beginning of each slot, each SU performs spectrum sensing to identify channel availabilities. The sensing duration has an uniform value equal to $\tau$, where $\tau<\delta$ (Fig. 2). Further, the sensing is assumed to be sequentially performed on $M$ channels one by one ${ }^{1}$. The sensing result is assumed to be imperfect with probability $\epsilon$, where $\epsilon \in(0,1)$. The imperfection may be due to factors like sensing-duration limitation and hardware sensitivity.

Once SU identifies a set of available channels, it can randomly select one of them for data transmission. Since multiple SUs may want to use the same channel in the same slot, this may lead to competition among them. To solve this

\footnotetext{
${ }^{1}$ For small number of channels, the sequential sensing is a major approach in recent studies [11]
}

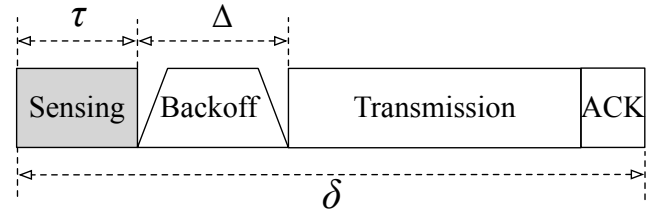

Fig. 2. CSMA/CA based protocol for SUs transmission.

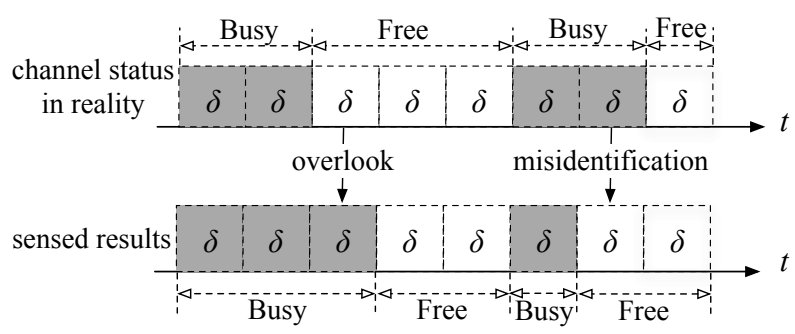

Fig. 3. Two sensing errors: overlook and misidentification.

problem, a CSMA/CA based protocol is suggested for SUs. This protocol is briefly described in the following (Fig. 2).

In a slot, after finishing the spectrum sensing, SU first selects an available channel if it exists. Then, SU generates a random backoff time $\Delta$, the value of which is uniformly selected in $\{0, w, 2 w, \ldots, C w\} . C$ is an integer, $w$ is called unit size of contention window, and the value of $C w$ is less than $(\delta-\tau)$. Further, when the backoff time expires, SU may detect the existence of other SUs signal ${ }^{2}$. If no other SU has started transmission in the selected channel, the particular SU transmits data to S-AP in the the remaining slot duration $(\delta-\tau-\Delta)$. Otherwise, SU keeps silence until next slot. Moreover, a successful transmission can be acknowledged by $\mathrm{S}$-AP at the end of the slot. For the simplicity of analysis, the duration of receiving acknowledgment message from S-AP is assumed to be zero.

\section{Sensing Errors and Transmission Collision}

Due to imperfect spectrum sensing, two different types of errors may occur in sensing results: 1) a free channel is sensed to be busy (so-called overlook), and 2) a busy channel is sensed to be free (so-called misidentification), as shown in Fig. 3. Overlook error leads to the overlooking of transmission opportunity in available channels. When misidentification error occurs, SU may select a busy channel. Further, by performing signal detection after backoff time, SU may know about the existence of PU signal in the selected channel, and thus it keeps silence until next slot. Since SU may be not able to differentiate the PU signal from other SU signals, it may obtain incorrect information that another SU is using the selected channel.

On the other hand, the CSMA/CA based protocol may lead to transmission collision among SUs. That is, two or

\footnotetext{
${ }^{2}$ As described in [6], the detection usually operates at a much higher signal strength than spectrum sensing, and thus the result is assumed to be perfect without any error.
} 


$$
\begin{gathered}
D_{m, n}(i)=\left\{\begin{array}{cc}
1, & {\left[\Delta_{m, n}(i)=\min \left\{\Delta_{m, 1}(i), \Delta_{m, 2}(i), \ldots, \Delta_{m, N}(i)\right\} \mid A_{m}(i)=0, \Delta_{m, n}(i) \neq+\infty\right]} \\
0, & {\left[\Delta_{m, n}(i)>\min \left\{\Delta_{m, 1}(i), \Delta_{m, 2}(i), \ldots, \Delta_{m, N}(i)\right\} \mid A_{m}(i)=0, \Delta_{m, n}(i) \neq+\infty\right]} \\
\operatorname{or}\left[A_{m}(i)=1, \Delta_{m, n}(i) \neq+\infty\right]
\end{array}\right. \\
\Omega(H)=\sum_{i=1}^{H} \sum_{n=1}^{N}\left[B\left(\delta-\tau-\Delta_{m, n}(i)\right) D_{m, n}(i) T_{m, n}(i) \mid \Delta_{m, n}(i) \neq+\infty\right] \\
X(H)=\sum_{i=1}^{H} \sum_{n=1}^{N}\left[B\left(\delta-\tau-\Delta_{m, n}(i)\right) D_{m, n}(i)\left(1-T_{m, n}(i)\right) \mid \Delta_{m, n}(i) \neq+\infty\right] \\
Y(H)=\sum_{i=1}^{H} \sum_{n=1}^{N}\left[B\left(\delta-\tau-\Delta_{m, n}(i)\right)\left(1-D_{m, n}(i)\right) A_{m}(i) \mid \Delta_{m, n}(i) \neq+\infty\right]
\end{gathered}
$$

more SUs may select the same free channel in a slot. If these SUs generate the same backoff time, they can start transmitting at the same time when backoff time expires. Subsequently, transmission collision among them happens, and thus they experience unsuccessful transmission by missing acknowledgment message from S-AP.

By jointly taking into consideration sensing errors, SUs competition and SUs transmission collision, a channel usage model is presented in the next subsection.

\section{Channel Usage Model}

Consider that the PU activity changes at discrete time points $\left\{t_{0}, t_{0}+\delta, t_{0}+2 \delta, \ldots, t_{0}+H \delta\right\}$. Let $h_{i}$ denote the time interval $\left[t_{0}+(i-1) \delta, t_{0}+i \delta\right]$, where $i=1,2,3, \ldots, H$. Let $A_{m}(i)$ denote the availability of channel $c_{m}$ in slot $h_{i}$, where $c_{m} \in$ $\left\{c_{1}, c_{2}, \ldots, c_{M}\right\} . A_{m}(i)$ is given by:

$$
A_{m}(i)= \begin{cases}1, & c_{m} \text { is actually free } \\ 0, & c_{m} \text { is actually busy }\end{cases}
$$

Let $\Delta_{m, n}(i)$ denote the possible backoff time used by SU $s_{n}$ in channel $c_{m}$ within slot $h_{i}$, where $s_{n} \in\left\{s_{1}, s_{2}, \ldots, s_{N}\right\}$. If channel $c_{m}$ is selected by SU $s_{n}$ in slot $h_{i}, \Delta_{m, n}(i)$ is in value interval $[0, C w]$. Otherwise, $\Delta_{m, n}(i)=+\infty$.

Further, let $D_{m, n}(i)$ denote the decision on whether or not SU $s_{n}$ can transmit data in the selected channel $c_{m}$ within slot $h_{i}$. The decision result is given by using CSMA/CA based protocol. $D_{m, n}(i)=1$ means the case that $\mathrm{SU} s_{n}$ can start transmission when its backoff time expires. Oppositely, $D_{m, n}(i)=0$ means the case that $\mathrm{SU} s_{n}$ should keep silence after backoff time expires. The numerical expression of $D_{m, n}(i)$ is shown by equation (1).

Furthermore, let $T_{m, n}(i)$ denote the transmission status of SU $s_{n}$ in channel $c_{m}$ within slot $h_{i}$, and it is:

$$
T_{m, n}(i)= \begin{cases}1, & \text { successful } \\ 0, & \text { unsuccessful due to collision }\end{cases}
$$

For the time interval $\left[t_{0}, t_{i}+H \delta\right]$, let $\Omega(H)$ denote the total throughput successfully used by SUs, and let $X(H)$ denote the total throughput wasted by SUs due to transmission collision among them. $\Omega(H)$ and $X(H)$ are computed with equations (2) and (3), respectively. Moreover, according to subsection II-B, the misidentification error on channel availability may make a SU select a busy channel. Although SU can keep silence when it finds the PU signal, this raises a wasting case that this SU may miss the transmission opportunity in another available channel. Let $Y(H)$ denote the total throughput wasted by SUs due to misidentification error. This is computed with equation (4).

Compared with SUs random channel selection, our goal is to develop a new spectrum decision strategy that can optimize SUs throughput $\Omega(H)$ in the presence of sensing errors, SUs competition and SUs transmission collision. This strategy is based on the channel usage prediction, which is presented in the following sections.

\section{Channel Usage Prediction}

\section{A. Channel Usage State}

To predict the channel usage, a new parameter is introduced, which is called Channel Usage State (CUS). CUS is used to represent the channel utilization observed at the SU side. It has three states, denoted by three symbols $\phi, S$, and $P$, respectively. For a SU $s_{n}$ in channel $c_{m}$ within slot $h_{i}$, the meanings of states $\phi, S$, and $P$ are as follows.

State $\phi$ means a situation when no other active users (PU or $\mathrm{SU})$ are identified by SU $s_{n}$. It includes two different cases:

- SU $s_{n}$ senses channel $c_{m}$ to be free, but $s_{n}$ does not select $c_{m}$ to transmit data.

- SU $s_{n}$ accomplishes a successful transmission in channel $c_{m}$ within slot $h_{i}$, i.e., $A_{m}(i)=D_{m, n}(i)=T_{m, n}(i)=1$.

State $S$ means a situation when SU $s_{n}$ has detected the signal from other active users. It includes three different cases:

- SU $s_{n}$ senses channel $c_{m}$ to be free, but it is actually occupied by PU, i.e., $A_{m}(i)=D_{m, n}(i)=0$.

- Channel $c_{m}$ is actually free, but SU $s_{n}$ 's backoff time is not the smallest one in slot $h_{i}$, i.e., $A_{m}(i)=1$ and $D_{m, n}(i)=0$.

- SU $s_{n}$ 's transmission collides with other SUs, i.e., $A_{m}(i)=D_{m, n}(i)=1$ and $T_{m, n}(i)=0$. 
TABLE I

AN EXAMPLE OF CUS HISTORY OVER 20 SLOTS.

\begin{tabular}{|c|c|c|c|c|c|c|c|c|c|c|c|c|c|c|c|c|c|c|c|c|}
\hline Slot & $h_{1}$ & $h_{2}$ & $h_{3}$ & $h_{4}$ & $h_{5}$ & $h_{6}$ & $h_{7}$ & $h_{8}$ & $h_{9}$ & $h_{10}$ & $h_{11}$ & $h_{12}$ & $h_{13}$ & $h_{14}$ & $h_{15}$ & 16 & $h_{17}$ & $h_{18}$ & $h_{19}$ & $h_{20}$ \\
\hline CUS & $\phi$ & $P$ & $\phi$ & $\phi$ & $S$ & $P$ & $\phi$ & $P$ & $\phi$ & $\phi$ & $\phi$ & $P$ & $S$ & $S$ & $\phi$ & $P$ & $P$ & $\phi$ & $\phi$ & $\phi$ \\
\hline
\end{tabular}

TABLE II

DECODED CONTEXTS

\begin{tabular}{|l|l|l|l|l|l|l|l|l|l|l|}
\hline$\phi$ & $P$ & $\phi \phi$ & $S$ & $P \phi$ & $P \phi \phi$ & $\phi P$ & $S S$ & $\phi P P$ & $P P$ & $\phi \phi \phi$ \\
\hline
\end{tabular}

State $P$ means a situation when $\mathrm{SU} s_{n}$ senses channel $c_{m}$ to be busy. Thus, channel $c_{m}$ is not a candidate for channel selection by $\mathrm{SU} s_{n}$.

\section{B. CUS History}

Consider that the CUS observation on a channel is carried out by a SU in consecutive slots $\left\{h_{1}, h_{2}, \ldots, h_{L}\right\}$. The observation results constitute a CUS history, where $L$ is called history length. Taking an example of $L=20$, this means that SU obtains 20 observation results, as shown in the example in Table I.

In the table, we are interested in two different types of statistics. The first one is about the respective occurrence probabilities of three states in the 20 slots. Since state $P$ occurs 6 times and state $S$ occurs 3 times, the occurrence probabilities of states $S$ and $P$ can be simply computed as $6 / 20=30 \%$ and $3 / 20=15 \%$, respectively. The second type of statistics is about the state change of CUS within two consecutive slots. For instance, in slots $h_{5}$ and $h_{6}$, the states of CUS are $S$ and $P$, respectively. The state change of CUS from slot $h_{5}$ to slot $h_{6}$ is denoted by $S \rightarrow P$. Clearly, the state change of $S \rightarrow P$ is different from the one of $P \rightarrow S$, because their state changes have opposite orders in time domain.

By learning from CUS history, SUs are able to know in advance the future channel availabilities. However, it is difficult to precisely predict in which state the CUS on a channel is expected to be. Thus, we instead focus on predicting the probability of every CUS state occurring in the near future.

\section{Predictor Selection}

The Markov family of predictors is considered to conduct prediction. In [9], [12], [13], the authors report on studies done on different Markov process based predictors like, e.g., Prediction by Partial Match (PPM), Lempel-Ziv78 (LZ78), Probabilistic Suffix Tree (PST), Context Tree Weighting (CTW). For these predictors, the prediction is based on the historical information over a specific time period. This information is commonly called history while the number of recording information is called storage length.

In [12], the authors report on a comparison of the performances of the above mentioned predictors. Various metrics are considered, e.g., storage length, number of alternative symbols, Markov class (variable or fixed), computation efficiency and computation complexity. The study shows that LZ78 is robust when a few alternative symbols exist in the history and it is preferable for prediction computation under moderate storage length. Moreover, the authors of [9] report on the study of the advanced algorithm of LZ78 (called LeZi-update scheme) for mobility purposes. By using it, the uncertainty associated with the location of a mobile can be predicted with a high degree of accuracy given its movement history. The movement history considers both the occurrence of visited areas and the transitions of different areas over time.

In our work, the CUS history covers only three symbols $\phi, S, P$ and the corresponding building has a similar manner with the movement history presented in [9]. Subsequently, we use the LeZi-update scheme to do CUS prediction.

\section{PREDiCTION IMPLEMENTATION}

The procedure of doing LeZi-update based prediction consists of two parts: parsing history and prediction computing. The first part is to parse the CUS history into different phrases and contexts. A phrase or a context is the sequence of one or more symbols from the set $\{\phi, S, P\}$. The phrases and the contexts are obtained by using encoding and decoding algorithms, respectively. The second part is to model a digital tree based upon the parsed contexts, and thus the prediction computing is performed.

\section{A. Parsing CUS History}

For the CUS history " $\phi P \phi \phi S P \phi P \phi \phi \phi P S S \phi P P \phi \phi \phi ”$, the encoding algorithm is started by considering the first symbol $\phi$. Since there is no phrase before this consideration, " $\phi$ " is determined as the first phrase. There are two rules after a phrase has been determined:

- rule-1: consider the next symbol that is following this phrase in the history;

- rule-2: if this phrase has been determined before, consider the combination of it and the next symbol.

According to the two rules, we show in Fig 4 the ten steps of determining different phrases. Consequently, " $\phi P \phi \phi S P \phi P \phi \phi \phi P S S \phi P P \phi \phi \phi$ " is encoded as " $\phi$ ", " $P$ ",

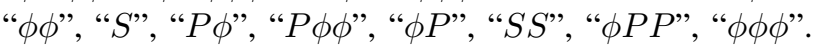

The decoding algorithm is to acquire the different contexts that have occurred in the encoded phrases. Taking a phrase " $\phi P P$ " for example, we say that its contexts are " $\phi$ ", " $P$ ", " $\phi P$ ", " $P P$ " and " $\phi P P$ ". By decoding all encoded phrases, we show all decoded contexts in Table II.

\section{B. Prediction Computing}

First, we use the decoded contexts to model a digital tree. The root of this tree is called level- 0 node with the meaning 
TABLE III

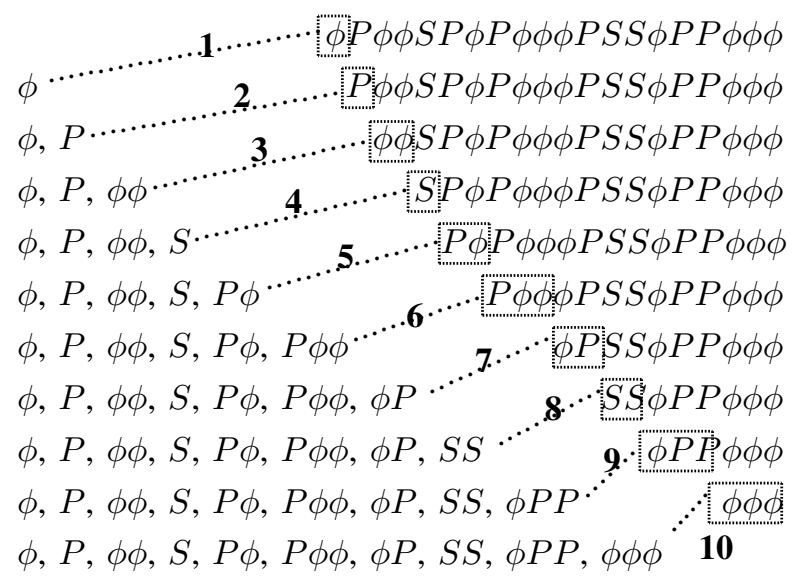

Fig. 4. Ten encoding steps.

of null context. We let the notation $\Lambda$ denote the root. The symbols that are the first symbol in the decoded contexts, are un-repeatedly modeled as the leaves of the root. The leaves of the root are called level-1 nodes. The symbols that are the second symbols in the decoded contexts are un-repeatedly modeled as the leaves of every level-1 node. The leaves of level-1 nodes are called level-2 nodes. It is clear that different level-1 nodes may have different leaves. Similarly, we can obtain the leaves of level-2 nodes, which are called level-3 nodes. Due to the null property of $\Lambda$, we consider $\Lambda$ as a leaf of any node in any level of the tree. We show the modeled digital tree in Fig 5.

By observing the digital tree, we need to find out all different tree branches, which are arising from the the root, e.g., two different branches " $(\Lambda-) \phi$ " and " $(\Lambda-) \phi \phi \phi$ ". Then, we count the occurrence time of every branch in all decoded contexts. For example, in context " $\phi \phi \phi$ ", the branch " $(\Lambda-) \phi$ " occurs three times, while the branch " $(\Lambda-) \phi \phi$ " occurs twice. Moreover, for a particular branch, we additionally mark the number of its occurrence time in the end node of the branch. Since the branch " $\phi P P$ " occurs once in all decoded contexts, we mark the number " 1 " with the level-3 node " $P$ ", as shown in Fig. 5.

By using the modeled digital tree, we do the prediction computing. The computation is based on a blending strategy, which is used in the prediction by partial match (PPM) scheme [15]. Further, the computation relies on the pre-determined order of Markov model. In [14], the authors studied various order Markov models by comparing prediction accuracy, coverage, model complexity and model size. Similar to [9], in our work we adopt the order-2 Markov model for prediction computing. The detailed computation process is as follows.

For the CUS history " $\phi P \phi \phi S P \phi P \phi \phi \phi P S S \phi P P \phi \phi \phi$ ”, we consider " $\Lambda$ " as the order- $O$ conditional event for prediction computing. We also consider the last symbol " $\phi$ " and two consecutive symbols of suffix " $\phi \phi$ " as order-1 and order2 conditional events, respectively. Further, by observing the
ALL PATHS OCCURRING UNDER CONDITIONAL EVENTS

\begin{tabular}{|c|cc|ccc|}
\hline \multicolumn{7}{|c|}{ Channel a : three conditional events } \\
\hline under $\phi \phi$ & \multicolumn{2}{|c|}{ under $\phi$} & \multicolumn{3}{c|}{ under $\Lambda$} \\
\hline$\phi(1)$ & $\phi(3)$ & $P(1)$ & $\phi(5)$ & $S(2)$ & $P(4)$ \\
$\Lambda(3)$ & $\phi \phi(1)$ & $P P(1)$ & $\phi \phi(3)$ & $\phi P(1)$ & $P \phi(1)$ \\
& $\Lambda(5)$ & & $P P(2)$ & $S S(1)$ & $\phi \phi \phi(1)$ \\
& & & $\phi P P(1)$ & $P \phi \phi(1)$ & $\Lambda(1)$ \\
\hline
\end{tabular}

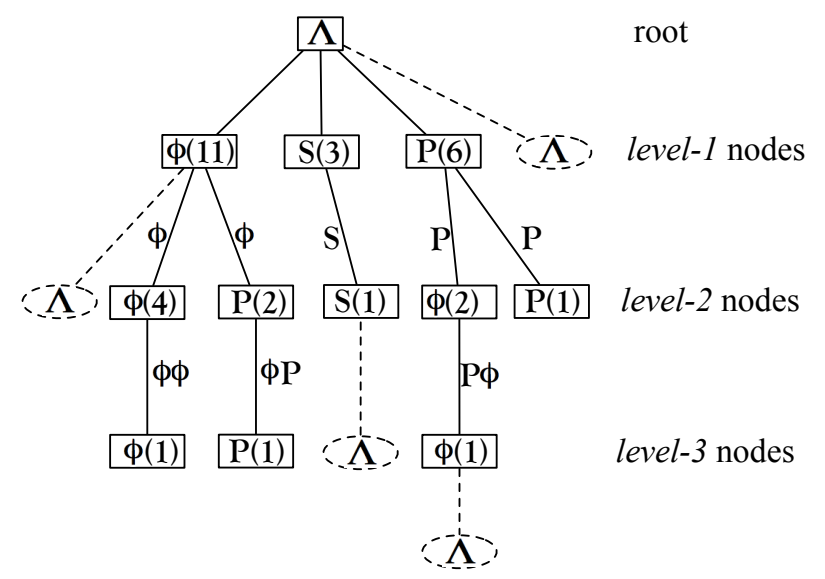

Fig. 5. The modeled digital tree.

digital tree in Fig. 5, we need to find out all different paths occurring under each of three conditional events. Here, a path can be thought of as a special branch that is arising from either the root or a leaf in the digital tree. The paths indicate the contexts that may occur in the near future. If a path occurs $\alpha$ times at different places in the tree, we denote it as $\operatorname{path}(\alpha)$. We report all found paths and their occurrence times in Table III.

We then compute the occurrence probability of each found path by using the formula:

$$
p_{r}(\text { path })=\frac{j_{2}}{z_{2}}+\frac{\gamma}{z_{2}} \cdot\left(\frac{j_{1}}{z_{1}}+\frac{\beta}{z_{1}} \cdot \frac{j_{0}}{z_{0}}\right)
$$

where

- $z_{0}, z_{1}, z_{2}$ : the frequencies of all possible paths (including $\Lambda$ ) occurring under order- 0 , order- 1 and order- 2 events, respectively.

- $j_{0}, j_{1}, j_{2}$ : the frequencies of a specific path occurring under order- 0 , order- 1 and order- 2 events, respectively

- $\beta, \gamma$ : the frequencies of $\Lambda$ occurring under order- 1 and order-2 events, respectively.

Moreover, both $\beta / z_{1}$ and $\gamma / z_{2}$ indicate the probability of a path falling back from an event to a lower order event, i.e., order- $1 \rightarrow$ order- 0 , order- $2 \rightarrow$ order- 1 .

Table III shows that 23 paths occur under $\Lambda, 11$ paths occur under $\phi$ and 4 paths occur under $\phi \phi$. According to equation (7), the occurrence probability of each path can be computed. All computed values are shown in Table IV. Moreover, for every computed value, we compute the occurrence probability of each of three states under the corresponding 
TABLE IV

PREDICTION COMPUTATION FOR CHANNEL A

\begin{tabular}{|c|c|c|c|c|c|c|c|}
\hline \multicolumn{8}{|c|}{ Channel a: $i / 4+3 / 4 \cdot(j / 11+5 / 11 \cdot k / 23)$} \\
\hline path & $i$ & $j$ & $k$ & $p_{r}($ path $)$ & $p_{r}(\phi)$ & $p_{r}(S)$ & $p_{r}(P)$ \\
\hline$\phi$ & 1 & 3 & 5 & 0.5287 & 0.5287 & 0.0000 & 0.0000 \\
\hline$S$ & 0 & 0 & 2 & 0.0296 & 0.0000 & 0.0296 & 0.0000 \\
\hline $\bar{P}$ & 0 & 1 & 4 & 0.1275 & 0.0000 & 0.0000 & 0.1275 \\
\hline$\phi \phi$ & 0 & 1 & 3 & 0.1126 & 0.1126 & 0.0000 & 0.0000 \\
\hline$\phi P$ & 0 & $\overline{0}$ & 1 & 0.0148 & 0.0074 & 0.0000 & 0.0074 \\
\hline$P \phi$ & 0 & 0 & 1 & 0.0148 & 0.0074 & 0.0000 & 0.0074 \\
\hline$P P$ & 0 & 1 & 2 & 0.0978 & 0.0000 & 0.0000 & 0.0978 \\
\hline$S S$ & 0 & 0 & 1 & 0.0148 & 0.0000 & 0.0148 & 0.0000 \\
\hline$\phi \phi \phi$ & 0 & $\overline{0}$ & 1 & 0.0148 & 0.0148 & 0.0000 & 0.0000 \\
\hline$\phi P P$ & 0 & 0 & 1 & 0.0148 & 0.0049 & 0.0000 & 0.0099 \\
\hline$P \phi \phi$ & 0 & 0 & 1 & 0.0148 & 0.0099 & 0.0000 & 0.0049 \\
\hline \multirow{2}{*}{\multicolumn{5}{|c|}{ Summation }} & $P_{\phi}$ & $P_{S}$ & $P_{P}$ \\
\hline & & & & & 0.6857 & 0.0444 & 0.2549 \\
\hline
\end{tabular}

path. For example, since one $\phi$ and two $P$ make up the path " $\phi P P$ " with probability 0.0148 , the individual probability of $(1 / 3 \times 0.0148) \simeq 0.0049$ is assigned to $\phi . P$ gets an individual probability of $(2 / 3 \times 0.0148) \simeq 0.0099$.

In Table IV, we sum all occurrence probabilities of $p_{r}(\phi)$ under every found path. The summation, denoted by $P_{\phi}$, indicates the probability of state $\phi$ occurring in the near future. Similarly, $P_{S}$ denotes the sum of all occurrence probabilities of $p_{r}(s)$ under every found path. $P_{P}$ denotes the sum of all occurrence probabilities of $p_{r}(P)$ under every found path. Furthermore, $P_{S}$ and $P_{P}$ indicate the probabilities of states $S$ and $P$ occurring in the near future, respectively.

\section{SPECTRUM Decision}

By predicting the probabilities of occurrence of $\phi, S$ and $P$, we can use them to estimate the channel availability for access by SUs. Since the three probabilities are independent from each other, this has promoted the need to combine them as a joint value. The joint value can be considered as one kind of decision criterion for spectrum decision.

To do the above mentioned combination, we use a fuzzy logic based comparison algorithm developed by Saaty [16]. The key procedure of this algorithm is to compare the importances of states $\phi, S$ and $P$ effecting the channel availability. The goal is to determine the combination weights of three probabilities.

Let $E_{\phi}, E_{S}$, and $E_{P}$ denote the effects of $\phi, S$ and $P$, respectively on the channel availability. The importance comparison of $E_{\phi}$ and $F_{S}$ is denoted by $E_{\phi} / E_{S}$. Similarly, let $E_{S} / E_{F}$ and $E_{F} / F_{\phi}$ denote the importance comparison of $E_{S}$ and $E_{F}$ and the importance comparison of $E_{F}$ and $E_{\phi}$, respectively. Meanwhile, we know that:

- For state $\phi$ : no PUs or other SUs using the channel is the best situation for a SU to use this channel.

- For state $S$ : several SUs sharing the same channel is a medium situation for another SU to access, since these SUs may compete for the channel utilization.

- For state P: PUs occupying the channel is the worst situation for a SU to access, since SUs can not use this channel to do transmission.
Similar to [17], we define one kind of comparison as follows:

$\Pi=\left[\begin{array}{ccc}E_{P} / E_{P} & E_{S} / E_{P} & E_{\phi} / E_{P} \\ E_{P} / E_{S} & E_{S} / E_{S} & E_{\phi} / E_{S} \\ E_{P} / E_{\phi} & E_{S} / E_{\phi} & E_{\phi} / E_{\phi}\end{array}\right]=\left[\begin{array}{ccc}1 & 3 & 5 \\ 1 / 3 & 1 & 3 \\ 1 / 5 & 1 / 3 & 1\end{array}\right]$

where $\Pi$ is called fuzzy-comparison matrix.

Matrix $\Pi$ has the eigen equation and the characteristic equation given by:

$$
\begin{array}{lll}
\Pi \Omega & = & \lambda V \\
\operatorname{et}(\Pi-\lambda I) & = & 0
\end{array}
$$

where $I$ is an unit matrix, $\lambda$ and $V$ are the eigen value and eigen vector of $\Pi$. The largest real eigen value corresponds to an eigen vector $V^{*}$ :

$$
V^{*}=\left\{v_{1}, v_{2}, v_{3}\right\} \simeq\{0.94,0.31,0.19\}
$$

The three coordinates of vector $V^{*}$ refer to combination weights of three probabilities $P_{\phi}, P_{S}$ and $P_{P}$, respectively. Thus, the joint value of the three probabilities is given by:

$$
\begin{aligned}
& P_{\phi} \cdot v_{1}+P_{S} \cdot v_{2}+P_{P} \cdot v_{3} \\
\simeq & 0.6857 \times 0.94+0.0444 \times 0.31+0.2549 \times 0.19 \\
\simeq & 0.7068
\end{aligned}
$$

This joint value indicates the numerical estimation of channel availability. In other words, the larger the joint value is, the higher the channel availability is expected (for SUs) in the near future. This means that the most appropriate channel in this particular case is the one associated with the largest joint value.

\section{Simulation Evaluation}

We have developed a simulator written in $\mathrm{C} / \mathrm{C}++$ [18]. Both the modeled CR network and the CUS prediction based spectrum decision are implemented in the simulator.

\section{A. Modification in Lezi-update Scheme}

In order to improve the computing efficiency, we did a modification in the LeZi-update scheme, as described in the following.

Given a CUS history " $\phi \phi \phi \phi \phi \phi \phi \phi \phi \phi P$ ", it is encoded as $\phi, \phi \phi, \phi \phi \phi, \phi \phi \phi \phi$ and $P$, by using the original encoding algorithm. We note that the phrase $\phi \phi \phi \phi$ contains four $\phi$. In more general cases, the encoded phrases may have more than four symbols. This results in five or more levels in the digital tree. The associated prediction computing becomes much more complex and time consuming. We therefore suggest an additional rule to the encoding algorithm:

- rule-3 the maximum number of the symbols in the determined phrase is limited to three.

According to rules 1, 2, and 3, " $\phi \phi \phi \phi \phi \phi \phi \phi \phi \phi P$ " is reencoded as $\phi, \phi \phi, \phi \phi \phi$ and $\phi P$, with low complexity for computing. 
TABLE V

PARAMETER SETTINGS.

\begin{tabular}{|l|l|l|}
\hline Parameter & Value & Description \\
\hline$B$ & 1 & Identical bandwidth of every channel \\
\hline$N$ & 3 & Number of SUs \\
\hline $1 / \mu_{p}$ & $0.3 \mathrm{~s}$ & Mean duration of state BUSY \\
\hline $1 / \lambda_{p}$ & $0.7 \mathrm{~s}$ & Mean duration of state FREE \\
\hline$\delta$ & $10 \mathrm{~ms}$ & Uniform duration of a slot \\
\hline$\tau$ & $1 \mathrm{~ms}$ & Uniform sensing duration in a slot \\
\hline$\epsilon$ & $5 \%$ & Probability of imperfect sensing \\
\hline$w$ & $0.05 \mathrm{~ms}$ & Unit size of contention window \\
\hline$C$ & 20 & Maximum contention counter \\
\hline$L$ & 30 & Length of CUS history \\
\hline
\end{tabular}

\section{B. Simulation Scenarios}

It is important for simulation to capture the key characteristics of CUS prediction based spectrum decision. They are: (i) how well the CUS prediction affects the access behavior of SUs, and (ii) how well the number of channels affects the performance of CUS prediction. To address these two characteristics, we consider four simulation sets, each with different channel number $N$ from the set $\{2,4,6,8\}$.

Every simulation set has two different scenarios, which are called random and prediction scenarios. These two scenarios correspond to random channel access and CUS prediction based spectrum decision, respectively. The random channel access means that SU randomly selects one of the available channels to do transmission.

Moreover, to perform an adequate statistical analysis, each simulation scenario was run 50 times and each simulation run was over 10000 s simulation time. The setting of other parameters is shown in Table $\mathrm{V}$.

\section{Results and Discussion}

Based on equations (2), (3) and (4), let $E[\Omega(H)], E[X(H)]$ and $E[Y(H)]$ denote the average value of $\Omega(H), X(H)$ and $Y(H)$ on 50 simulation runs, respectively. The simulation results are shown in Figs. 6, 7 and 8, together with the 95\% confidence interval.

For the fixed channel number $M$, Fig. 6 shows that $E[\Omega(H)]$ under CUS scenario is larger than the one under random scenario. This means that the throughput of SUs successful transmission can be improved by using CUS prediction based spectrum decision. Further, for either random or CUS scenario, $E[\Omega(H)]$ increases with $M$. This is because the larger number of free channels that can be provided for SUs. Thus, SUs can successfully transmit more data to S-AP when $M$ increases.

For the fixed $M$, Fig. 7 shows that $E[X(H)]$ under CUS scenario is also larger than the one under random scenario. This is because CUS prediction may make SUs concentrate on the same available channels. As a result, the competition among SUs is enhanced by using the CUS prediction based spectrum decision. However, when $M$ increases, SUs may use much more different channels, and thus the problem of SUs transmission collision is alleviated. This is addressed by

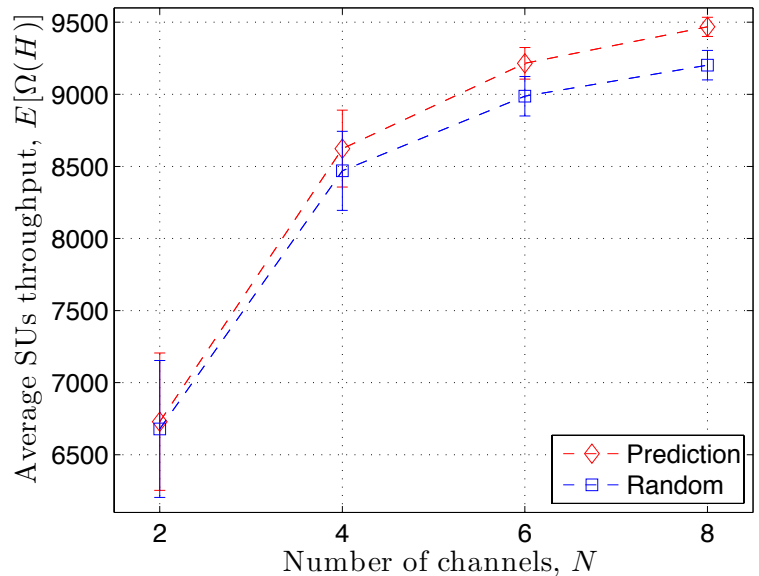

Fig. 6. Average throughput of SUs successful transmission, $E[\Omega(H)]$.

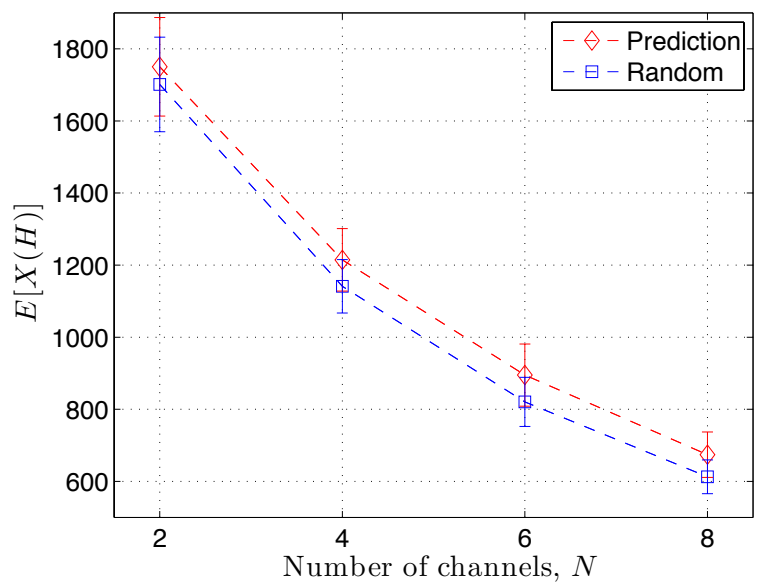

Fig. 7. Average wasted throughput due to SUs transmission collision, $E[X(H)]$.

that $E[X(H)]$ decreases with $M$ for either random or CUS scenario.

As observed in Fig. 8, for the same $M, E[Y(H)]$ under CUS scenario is smaller than the one under random scenario. Moreover, $E[Y(H)]$ increases with $M$ for random scenario, whereas $E[Y(H)]$ decreases with $M$ for CUS scenario. The reason for these is as follows. Consider that the sensing imperfection probability $\epsilon$ is a constant value. When the number of channels increases, SUs may misidentify much more available channels that are actually busy. Thus, the probability of selecting busy channels is increased for SUs under random scenario. While under CUS scenario, the occurrence probability of three states $P, \phi$ and $S$ are predicted. Further, the decision maker gives lower combination weights to states $P$ and $S$ than state $\phi$. Hence, SUs can be good at looking for the actually free channels by using CUS prediction based spectrum decision. As a consequence, for SUs under CUS scenario, the probability of selecting busy channels decreases with $M$ 


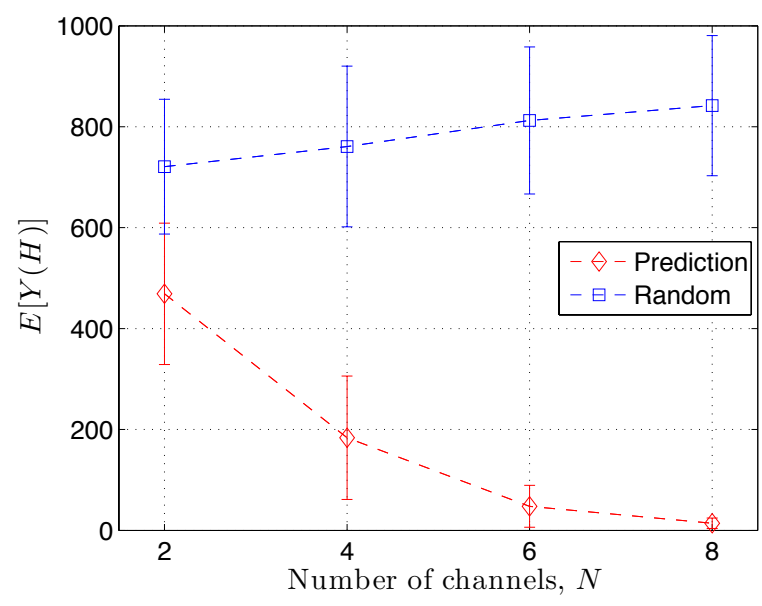

Fig. 8. Average wasted throughput due to SUs misidentification error, $E[Y(H)]$.

\section{CONCLUSION}

A realistic channel usage model was studied in the presence of sensing error, SUs competition and SUs transmission collision. Based on this model, a new parameter was introduced, which is called Channel Usage State (CUS). CUS is used to represent the channel utilization observed at the SU side. The observation results are composed of three situations, namely, no other active users (PU or SU), experiencing a successful transmission and being aware of other active users. By using the LeZi-update scheme, the occurrence probabilities of the three situations were predicted. A fuzzy logic based comparison algorithm was adopted to combine three probabilities as a joint value. The spectrum decision was done in a manner that the largest joint value indicates the most available channel. Simulation experiments were carried out to evaluate the performance and effectiveness of CUS prediction based spectrum decision. The results demonstrated that the CUS prediction based spectrum decision outperforms the random channel access. Future work is to compare the performance of CUS prediction with other Markov process based algorithms for doing spectrum decision.

\section{REFERENCES}

[1] G. Staple and K. Werbach, The end of spectrum scarcity, IEEE Spectrum, vol. 41, no. 3, pp. 48-52, Mar. 2004.

[2] I.F. Akyildiz, W.Y. Lee, M.C. Vuran, and S. Mohanty, "NeXt generation/dynamic spectrum access/cognitive radio wireless networks: a survey", Elsevier Computer Networks, vol. 50, pp. 2127-2159, 2006.

[3] W.Y. Lee, K.R. Chowdhury, and M.C. Vuran, "Spectrum Sensing Algorithms for Cognitive Radio Networks", Cognitive Radio Networks, pp. 3-35, CRC Press, Taylor \& Francis Group, 2009.

[4] B. Wang, Z. Ji, and K.J.R. Liu "Primary-Prioritized Markov Approach for Dynamic Spectrum Allocation", IEEE Transaction on Wireless Communication (TWC), vol. 8, no. 4, pp. 1854-1865, April 2009.

[5] Q. Zhao, L. Tong, A. Swami, and Y. Chen, "Decentralized cognitive MAC for opportunistic spectrum access in ad hoc networks: a POMDP framework", IEEE Journal on Selected Areas in Communications (JSAC), vol. 25, no. 3, pp. 589-600, April 2007.

[6] A.T. Hoang, D.T.C. Wong, and Y.C. Liang, "Design and Analysis for an 802.11-based Cognitive Radio Network", IEEE Wireless Communications and Networking Conference (WCNC), Budapest, Hungary, April 2009.

[7] L.F. Lai, H.E. Gamal, H. Jiang, and H.V. Poor, "Cognitive Medium Access: Exploration, Exploitation, and Competition", IEEE Transactions on Mobile Computing (TMC), vol. 10, no. 2, pp. 239-253, February 2011.

[8] L. Akter, B. Natarajan and C. Scoglio, "Spectrum Usage Modeling and Forecasting in Cognitive Radio Networks", Cognitive Radio Networks, pp. 37-60, CRC Press, Taylor \& Francis Group, 2009.

[9] A. Bhattacharya and S.K. Das, "LeZi-Update: An Information-Theoretic Framework for Personal Mobility Tracking in PCS Networks", Wireless Networks, vol. 8, no. 2/3, pp. 121-135, 2002

[10] A.O. Popescu, D. Erman, M. Fiedler, A.P. Popescu, and D. Kouvatsos, "A middleware framework for communication in cognitive radio networks", International Congress on Ultra Modern Telecommunications and Control Systems (ICUMT), Moscow, Russia, October 2010.

[11] X.Y. Wang, A. Wong, and P.H. Ho, "Stochastic Medium Access for Cognitive Radio Ad Hoc Networks", IEEE Journal on Selected Areas in Communications (JSAC), vol. 29, no. 4, pp. 770-783, April 2011.

[12] D. Katsaros and Y. Manolopoulos, "Prediction in Wireless Networks by Markov Chains", IEEE Wireless Communications, vol 16, no. 2, pp. 56-63, April 2009.

[13] S.R. Ngoga, D. Erman, and A.P. Popescu, "Predictive Models for Seamless Mobility, Sixth International Working Conference on. Performance Modeling and Evaluation of Heterogeneous Networks (HETNETs), Zakopane, Poland, January 2010.

[14] M. Deshpande and G. Karypis, "Selective Markov Models for Predicting Web Page Accesses", ACM Transactions on Internet Technology, vol. 4, no. 2, pp. 163-184, 2004.

[15] J.G. Cleary and I.H. Witten, "Data Compression Using Adaptive Coding and Partial String Matching", IEEE Transactions on Communications, vol. 32, no. 4, pp. 396-402, April 1984.

[16] T.L. Saaty, "Exploring the Interface Between Hierarchies, Multiple Objectives and Fuzzy Sets", Fuzzy Sets and Systems, vol. 1, issue 1, pp. 57-68, 1978.

[17] E. Rakus-Andersson, "The Choice of Optimal Medicines", Fuzzy and Rough Techniques in Medical Diagnosis and Medication, Berlin Heidelberg: Springer-Verlag, 2007.

[18] Y. Yao, "A Spectrum Decision Support System for Cognitive Radio Networks", Licentiate Thesis, Blekinge Institute of Technology (BTH), Sweden, May 2012. 